therapy is only an adjunct to more active rehabilitation. We have found it particularly useful in the early mobilization of stiff joints-e.g., fingers and shoulders. In patients so severely injured as to be discharged from the Services-in which more energetic treatment is ruled out by the disability-we have found occupational therapy of considerable value. In reconstructional surgery of the severely injured it will no doubt prove a useful preliminary to the later vocational training.

\section{Medical Staff}

The work in the occupational-therapy department at the auxiliary hospitals and at the rehabilitation annexe is supervised by one member of the staff who has taken a particular interest in it and who sees the patients regularly. We feel that in the absence of a full-time rehabilitation officer this supervision is best done by one of the surgical team, who is thereby enabled to establish continuity of treatment. We feel also that in some ways he has an advantage over the full-time rehabilitation officer, for the role of the medical man in rehabilitation, apart from supervising and detailing treatment, is largely that of a medical whip and a mental poultice. The patients are, on the whole, more willing to be spurred on by the surgeon who has already had them under his care. The work requires patience, tact, and driving power, and one is more able to drive up to the patient's limit if one has already gained his confidence and also has a clear conception of the pathology of each case. No doubt the time will come when all rehabilitation will be carried out under the care of full-time officers; but we feel that their work should be closely co-ordinated and supervised by those responsible for the previous surgical treatment.

This development in rehabilitation work has been made practicable by the co-operation of the Regional Hospital Officer, Major-Gen. R. W. D. Leslie, and thanks are due to him and his deputy, Dr. A. Alan Forty, for their willingness to give every assistance in the establishment and equipment of the rehabilitation annexe and in the co-ordination of the work in the auxiliary hospitals.

REFERENCES

Nicoll, E. A. (1941). British Medical Journal, 1, 501.

Watson-Jones, R. (1942). Ibid., 1, 403.

\section{A UNIVERSAL APPARATUS FOR PERORAl. INTRATRACHEAL ANAESTHESIA}

\author{
BY
}

GRAHAM HUMBY, M.R.C.S.

E.M.S. Plastic Surgeon; E.M.S. Surgeon to the Hospital for Sick Children

WITH THE ANAESTHETIST'S POINT OF VIEW

BY

MARGARET HAWKSLEY, M.B.

E.M.S. Anaesthetist, Base Hospital, Hemel Hempstead

There is no branch of surgery in which the surgeon is more intimately associated with the anaesthetist than in oral surgery, and more particularly cleft-palate operations ; with this excuse I take the liberty of expressing my ideas on the subject.

Intratracheal anaesthesia for oral surgery is unsatisfactory, because there is no apparatus universally applicable to the wide variety of common operations. What are the desiderata for a peroral intratracheal anaesthetic in an infant who may have to be anaesthetized for an hour or so? Infants are quoted because they are the most difficult subjects to anaesthetize, and it was upon 400 hare-lip and cleft-palate cases that the universal apparatus has been evolved.

\section{Desiderats}

Mechanical.-(1) It must be possible to use the apparatus with or without a Davis gag-i.e., for hare-lip or cleft-palate cases. (2) It must stay in position without packing once it is introduced. (3) It must not be kinked or squashed. (4) The intratracheal portion must be of rubber to produce the minimum tracheitis or laryngitis. (5) It must be easily sterilized. (6) The apparatus must be able to fit snugly into the gag without pressing it on the lower lip.

Physiological.-(1) An infant must be kept in the second stage of anaesthesia indefinitely. Contrary to the usual teaching, gas-and-oxygen is the perfect anaesthetic in infants so long as the percentage of the gases is controlled. All the cases had nitrous oxide after induction, and among them were cases of protruding premaxilla hare-lip in infants 1 month old in which the premaxilla was replaced in addition to the bilateral lip repair-a long and shock-producing operation. There was no operative or anaesthetic mortality. (2) Carbon dioxide must be expelled as near to the lungs as is possible because infants will not tolerate a raised alveolar carbon dioxide percentage. (3) No positive pressure or rebreathing must be allowed in infants because of the coincident raised blood pressure and respiration depth.

\section{Description of the Apparatus}

The universal apparatus to be described fulfils these conditions. It is made in two sizes,--for adults and children respectively, and consists of an intratracheal part (Fig. 1, A) and a chin-piece (Fig. 1, B).

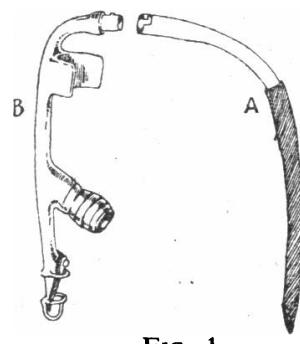

Fig. 1

Intratracheal Part.-This consists of a rubber tube pressed firmly on to the serrated end of a curved metal tube. Three sizes and curves of the metal portion are made for children, and they correspond to sizes 00,0 , and 1 Magill nasal tubes. The rubber portions are $2 \frac{1}{2}$ in., $2 \frac{3}{4}$ in., and 3 in. long respectively, and are used on children from 1 month to 4 years old. The adult intratracheal sizes are made to demand; the chinpiece corresponds to a No. 10 Magill. The intratracheal portion is passed by means of a laryngoscope and united to the chin-piece by a bayonet joint which works through a right-angle, ensuring that it cannot be lost down the throat.

Chin-piece.-The.inspiratory opening is placed under the chin at an angle to the main tube, promoting easy inspiration; it will be noticed that the inspiratory lumen is as large as the largest intratracheal tube. The expiratory opening is guarded by a thin rubber valve. In the adult model the valve can be removed and replaced by a length of rubber tubing or a metal cap should rebreathing or a closed circuit be desired. A curved chin-strap rests below the lower lip to prevent the apparatus from rotating, and this is fixed to the chin by adhesive strapping.

Fig. 2 shows how snugly the apparatus fits a Davis type gag: the only alteration necessary for the apparatus to fit a standard gag is the addition of a slot in the tongue depressor to allow the metal part of the tube to lie out of the way.

\section{THE ANAESTHETIST'S POINT OF VIEW}

The standard Boyle's gas-oxygen apparatus, as supplied by the Ministry of Health to Emergency Medical Service hospitals, has been used with this apparatus. The only expiratory valve employed is that which is attached to the chin-piece and already described.

Induction.-The infant, premedicated only with atropine, is induced on the operating table, the agent of choice being vinyl ether on an open mask. This provides a quick induction with ready relaxation of the jaw, the required depth being quickly reached and as quickly recovered from, and the laryngeal spasm so common with ethyl chloride is very rarely encountered.

Intubation.-The passing of the tube may present a few difficulties, particularly in infants of 4 weeks or so. A smallsize laryngoscope is used, and the tube is passed via the mouth by direct vision. In adults it is not always necessary actually to see the vocal cords when passing the tube if the epiglottis 
and oesophagus are clearly in view, but in the infant it is essential to make sure that the tip of the tube passes through the vocal cords, otherwise it may slip behind the larynx and down the oesophagus. To bring the cords clearly into view the shoulders are first raised by an assistant and then, when the epiglottis is seen, gently replaced on the table. It is advisable not to touch the epiglottis with the laryngoscope, as this may result in immediate spasm of the vocal cords. Attempts to pass the tube should not be made until conditions for so doing are at their best; second and third attempts are more difficult, and are harmful to the child. The infant should be in the third stage of anaesthesia, breathing quietly and easily; if the plane is not deep enough laryngeal spasm will probably be encountered, preventing passage of the tube and necessitating a second attempt.

Maintenance.-The gas and oxygen are turned on just beforehand and the apparatus laid to hand ready connected except for the bayonet joint. Once the tube is in situ and connected, gas and oxygen and sometimes two or three whiffs of ether only are required. The rate of flow is about half the rate usually necessary with ordinary intubation and expiratory valve. For a child 1 year old 2 litres of $\mathrm{N}_{2} \mathrm{O}$ and $1 / 2$ litre of $\mathrm{O}_{2}$ per minute are necessary. The anaesthesia lightens rapidly to the second stage with reappearance of the pharyngeal reflex, which the surgeon can evoke when he wishes to assess the adequacy of his operation. As the last stitches are being inserted the gas is turned off, and when the tube is removed the anaesthesia is light enough for the child to cry. and cough.

\section{Comparison with Other Methods}

Simple Oral Anaesthesia.-Comparison with other methods of anaesthesia, such as a hooked tube in the mouth or an attachment to tongue forceps delivering ether and oxygen, shows very obvious advantages. Without an endotracheal tube it is impossible to keep the child on a light even plane of anaesthesia. Either the child is lightly anaesthetized and by its gagging and moving impedes the surgeon or else it is deeply under, with consequent disadvantages to itself and greater risk of post-operative complications, formerly so common in this branch of surgery.

Magill Armoured Tube.-Advantages of the apparatus over the Magill unkinkable endotracheal tube are: (1) It stays in place.without a gag, a gag being unnecessary in the case of harelip operations, as the metal portion extends over the back of the tongue. The Magill tube is inclined to ride up even when packed, because of the metal spiral it contains. (2) It cannot wear out if used with a gag, as only the metal portion comes into contact with it. Fresh rubber ends can be fitted each time if necessary, as it is inadvisable to use tubes after they have become softened by boiling. (3) A fully controlled anaesthesia is obtained. The large-bore inlet allows gases to be drawn into the lungs as required by the child's respirations and exhaled easily by means of the simple valve close to the face. This is not the case with the Magill tube, in which gases under slight positive pressure are supplied by the smallbore inlet tube, no bag or expiratory valve being used: incidentally, omission of a bag causes a waste of gas. Also, the funnel-shaped expiratory opening of the armoured tube, perhaps elongated with rubber tubing, may upset the gasoxygen percentages should the child increase its inspiratory effort from external causes. (4) Actual passing of the tube is slightly easier owing to its curve; with this apparatus the anaesthetist's right hand is at the side of the mouth and therefore does not obstruct the view of the glottis.

Magill Nasal Tube.-This interferes with the contraction of the nasopharyngeal sphincter and any manipulations that may be necessary with the nasal mucosa. If a rubber tube is used orally with a gag it is apt to be squashed or kinked by the tongue depressor either on the tongue or against the posterior pharyngeal wall.

Disadvantages of the Apparatus.-The disadvantages should be mentioned. The apparatus consists of three portions, and it may be argued that these could come apart; but if that possibility is borne in mind and the rubber tube is pushed on up to the upper limit of the serrations this should never happen: it is important to fit the right-sized rubber to the right-sized metal. In an earlier model the bayonet joint between the two main portions occasionally slipped undone, but the improved joint working through a right-angle precludes this possibility.

Comment

To sum up, then, this apparatus provides an even, light, controlled gas-oxygen anaesthesia. Once the apparatus is in position, a procedure which should take altogether from five to seven minutes, neither anaesthetist nor surgeon has any further worry: the child is round immediately the operation is finished, and there are no post-operative complications due to the anaesthetic. Although originally designed for use in children it is evident that the apparatus will be a valuable addition to the anaesthetist's outfit for all operations on the head and neck in adults.

Those who are interested in the practical application of the apparatus (made by Down Bros.) are welcomed at Hemel Hempstead Base Hospital on Tuesdays at 9.30 a.m.

\section{IMPETIGO CONTAGIOSA IN THE SERVICES WITH SPECIAL REFERENCE TO ITS TREATMENT WITH SULPHATHIAZOLE \\ BY}

\section{G. A. GRANT PETERKIN, M.B., B.Ch.}

Major, R.A.M.C. : Dermatological Specialist; formerly Assistant Physician, Skin Department, Royal Infirmary, Edinburgh AND

E. COLN JONES, M.B., B.S.

Lieut., R.A.M.C. : Graded Dermatologist

The treatment of dermatological cases in the Services is in many respects more satisfactory than in civil life, as the average contagious skin case, to prevent further infection in the unit, is admitted to hospital and up-to-date methods are available. Compared with those in civil practice, the cases of impetigo treated in the Services are on the average more severe, and a certain number proved resistant to treatment, many healing slowly, sometimes because of a persistent folliculitis and sometimes because of tiny reinoculations, causing small oozing lesions.

In a military hospital entirely for dermatological patients 1,432 cases were admitted during the five months from May 14 to Oct. 14, and 396 of these were impetigo, so that there was ample opportunity of studying the action of drugs on this disease. A number of types were encountered, which for clinical purposes might be divided into the following groups: (1) Extensive, with excessive crusting (i.e., often the whole face affected). (2) Extensive, with moderate crusting. (3) Extensive, with small weeping crusted lesions (i.e., up to $1 \mathrm{~cm}$. diameter). (4) Mild-one or two areas. (5) Mixed: excessive crusts (indicating a streptococcal origin), with superficial vesicular lesions and perhaps folliculitis (staphylococcal). (6) Staphylococcal type (i.e., initial small bullae), followed by superficial crusting with slight inflammation, peripheral vesiculation, and residual pigmentation (Roxburgh, 1941). (7) A type of seborrhoeic skin characterized by small superficial erosions, and often associated with a retro-auricular dermatitis.

\section{Differential Diagnosis}

The differential diagnosis proved to vary somewhat from that in civil practice, and the following conditions seemed to be easily mistaken for impetigo.

1. Respirator Dermatitis.-Every case of this was referred as an impetigo, as the cheeks, chin, and brow were covered with golden-yellow crusts with marked exudation; the typical distribution, especially the involvement of the submental region, and the sharp demarcation of the lesions, followed by a positive patch test, established the diagnosis.

2. Sycosis.-The duration of the disease was often alone sufficient to make the diagnosis, without the clinical appearances, but some cases of impetigo of the beard region which had been present for several weeks were tending to drift gradually to a true sycosis.

3. Herpes Simplex.- In the crusted stage it was virtually impossible to distinguish this disease from a mild impetigo if an intelligent history was not obtainable. There seemed no doubt that some cases of extensive impetigo had begun as an infection of the original herpes. 